\title{
On Randić Energy of Coral Trees*
}

\author{
Xuan Zhao ${ }^{a}$, Yanling Shao ${ }^{b, \dagger}$, Yubin $\mathrm{Gao}^{b}$ \\ ${ }^{a}$ School of Data Science and Technology, North University of China, \\ Taiyuan, Shanxi 030051, P. R. China \\ ${ }^{b}$ School of Sciences, North University of China, \\ Taiyuan, Shanxi 030051, P. R. China
}

(Received December 28, 2021)

\begin{abstract}
Let $G$ be a simple and connected graph. A vertex $v_{i}$ is said to be pendent if $d_{G}\left(v_{i}\right)=1$, and its adjacent vertex is called a quasi-pendent vertex. Let $\mathcal{T}(n)$ be a set of trees of order $n$ with at most two quasi-pendent vertices of degree less than 4. We name $\mathcal{T}(n)$ the set of coral trees.

The Randić matrix of $G$, denoted by $R(G)$, is an $n \times n$ matrix whose $(i, j)$-entry is equal to $\frac{1}{\sqrt{d_{G}\left(v_{i}\right) d_{G}\left(v_{j}\right)}}$ if $v_{i} v_{j} \in E(G)$, and 0 otherwise. The Randić energy of $G$ is defined as

$$
\mathcal{E}_{R}(G)=\sum_{i=1}^{n}\left|\mu_{i}(G)\right|,
$$

where $\mu_{1}(G), \mu_{2}(G), \ldots, \mu_{n}(G)$ are the eigenvalues of $R(G)$.

In [I. Gutman, B. Furtula, S. B. Bozkurt, On Randić energy, Linear Algebra Appl. 442 (2014) 50-57], the authors conjectured that for a tree $T$ of order $n$, if $n$ is odd, then the maximum $\mathcal{E}_{R}(T)$ is achieved for $T$ being the $\left(\frac{n-1}{2}\right)$-sun; if $n$ is even, then the maximum $\mathcal{E}_{R}(T)$ is achieved for $T$ being the $\left(\left\lceil\frac{n-2}{4}\right\rceil,\left\lfloor\frac{n-2}{4}\right\rfloor\right)$-double sun.

In this work, we get the following results.

(1) For $T \in \mathcal{T}(n), \mathcal{E}_{R}(T) \leq \mathcal{E}_{R}\left(P_{n}\right)$.

(2) For a graph $G$, if $\mathcal{E}_{R}(G) \leq \mathcal{E}_{R}\left(P_{n}\right)$, then $G$ satisfies the conjecture.

(3) $\mathcal{T}(n)$ is a family of trees that satisfies the conjecture.
\end{abstract}

\section{Introduction}

Let $G$ be a simple and connected graph of order $n$ with vertex set $V(G)=\left\{v_{1}, v_{2}, \ldots, v_{n}\right\}$ and edge set $E(G)$. For $i=1,2, \ldots, n$, denote by $d_{G}\left(v_{i}\right)$ the degree of the vertex $v_{i}$ in $G$.

\footnotetext{
*Research supported by Shanxi Scholarship Council of China (No.201901D211227).

†Corresponding author. E-mail address: ylshao@nuc.edu.cn (Y. Shao).
} 
A vertex $v_{i} \in V(G)$ is said to be pendent if $d_{G}\left(v_{i}\right)=1$, and its adjacent vertex is called a quasi-pendent vertex of $G$. If a quasi-pendent vertex has degree less than 4, then we call it a small quasi-pendent vertex.

Let $\mathcal{T}(n)$ be the set of trees of order $n$ with at most two small quasi-pendent vertices. For each $T \in \mathcal{T}(n)$, we name it coral tree and say that $\mathcal{T}(n)$ is the set of coral trees.

The Randić index of a graph $G$ is defined as ( [13])

$$
\chi(G)=\sum_{v_{i} v_{j} \in E(G)} \frac{1}{\sqrt{d_{G}\left(v_{i}\right) d_{G}\left(v_{j}\right)}} .
$$

The Randić matrix of $G$, denoted by $R(G)([3,4,11])$, is an $n \times n$ matrix whose $(i, j)$ entry is equal to $\frac{1}{\sqrt{d_{G}\left(v_{i}\right) d_{G}\left(v_{j}\right)}}$ if $v_{i} v_{j} \in E(G)$, and 0 otherwise. The Randić energy of $G$ is defined as ( [4])

$$
\mathcal{E}_{R}(G)=\sum_{i=1}^{n}\left|\mu_{i}(G)\right|,
$$

where $\mu_{1}(G), \mu_{2}(G), \ldots, \mu_{n}(G)$ are the eigenvalues of $R(G)$.

Let $p \geq 0$. The tree $S u_{p}$ of order $n=2 p+1$, containing with $p$ pendent vertices, each attached to a vertex of degree 2 , and a vertex of degree $p$, is called the $p$-sun. Let $p, q \geq 0$. The tree $D S u_{p, q}$ of order $n=2(p+q+1)$, obtained from a $p$-sun and a $q$-sun, by connecting their central vertices, is called a $(p, q)$-double sun.

In [11], the authors proved that the star $S_{n}$ is the unique tree with minimal Randić energy over all trees, pointed out that for $n \geq 7$, the path $P_{n}$ is not the connected $n$-vertex graph with maximal Randić energy. They also presented the following conjecture about the trees with maximal $\mathcal{E}_{R}(T)$.

Conjecture 1.1 ( [11]) Let $T$ be a tree of order $n$. If $n$ is odd, then the maximum $\mathcal{E}_{R}(T)$ is achieved for $T$ being the $\left(\frac{n-1}{2}\right)$-sun. If $n$ is even, then the maximum $\mathcal{E}_{R}(T)$ is achieved for $T$ being the $\left(\left\lceil\frac{n-2}{4}\right\rceil,\left\lfloor\frac{n-2}{4}\right\rfloor\right)$-double sun.

In [9], the authors got the minimal Randić energy of trees with given diameter. In [8], the author showed that the generalized double suns of odd order satisfy Conjecture 1.1. In $[1,2]$, the authors presented some families of graphs that satisfy Conjecture 1.1. For more related research results, we refer to $[3,4,6,7,9,11,12]$.

In this paper, we get the following results.

(1) For $T \in \mathcal{T}(n), \mathcal{E}_{R}(T) \leq \mathcal{E}_{R}\left(P_{n}\right)$.

(2) For a graph $G$, if $\mathcal{E}_{R}(G) \leq \mathcal{E}_{R}\left(P_{n}\right)$, then $G$ satisfies Conjecture 1.1.

(3) $\mathcal{T}(n)$ is a family of trees that satisfies Conjecture 1.1 . 


\section{Preliminaries}

For a tree $T$, we use $\mathcal{M}_{k}(T)$ to denote the set of all $k$-matchings of $T$. If $e=v_{i} v_{j} \in E(T)$ and $\alpha_{k}=\left\{e_{1}, e_{2}, \ldots, e_{k}\right\} \in \mathcal{M}_{k}(T)$, then we denote $R_{T}(e)=R_{T}\left(v_{i} v_{j}\right)=\frac{1}{d_{G}\left(v_{i}\right) d_{G}\left(v_{j}\right)}$ and $R_{T}\left(\alpha_{k}\right)=\prod_{i=1}^{k} R_{T}\left(e_{i}\right)$, respectively.

Let $T$ be a tree of order $n$ with matrix $R(T)$. Then the Randic characteristic polynomial of $T$ can be written as ( [5])

$$
\phi_{R}(T, x)=|x I-R(T)|=\sum_{k=0}^{\left\lfloor\frac{n}{2}\right\rfloor}(-1)^{k} b(R(T), k) x^{n-2 k},
$$

where $b(R(T), 0)=1$, and $b(R(T), k)=\sum_{\alpha_{k} \in \mathcal{M}_{k}(T)} R_{T}\left(\alpha_{k}\right)$ for $1 \leq k \leq\left\lfloor\frac{n}{2}\right\rfloor$.

Lemma 2.1 ( [9]) Let $T_{1}$ and $T_{2}$ be two trees of order $n$, and let their Randić characteristic polynomials be

$$
\phi_{R}\left(T_{1}, x\right)=\sum_{k=0}^{\left\lfloor\frac{n}{2}\right\rfloor}(-1)^{k} b\left(R\left(T_{1}\right), k\right) x^{n-2 k}, \quad \phi_{R}\left(T_{2}, x\right)=\sum_{k=0}^{\left\lfloor\frac{n}{2}\right\rfloor}(-1)^{k} b\left(R\left(T_{2}\right), k\right) x^{n-2 k},
$$

respectively. If $b\left(R\left(T_{1}\right), k\right) \leq b\left(R\left(T_{2}\right), k\right)$ for all $k \geq 0$, and there is a positive integer $k$ such that $b\left(R\left(T_{1}\right), k\right)<b\left(R\left(T_{2}\right), k\right)$, then

$$
\mathcal{E}_{R}\left(T_{1}\right)<\mathcal{E}_{R}\left(T_{2}\right)
$$

Lemma 2.2 ( $[\mathbf{1 0}])$ Let $T$ be a tree of order $n$. Then $\left|\mathcal{M}_{k}(T)\right| \leq\left|\mathcal{M}_{k}\left(P_{n}\right)\right|$ for $1 \leq k \leq$ $\left\lfloor\frac{n}{2}\right\rfloor$.

\section{$3 \quad$ Some operations}

Let $T$ be the tree as shown in Figure 1, where $T_{1}$ is a subtree of $T$ with $v_{0} \in V\left(T_{1}\right), t \geq 2$, and $d_{T}\left(v_{0}\right) \geq 3$. Let $T^{\prime}=T-\left\{v_{0} v_{2}, \ldots, v_{0} v_{t}\right\}+v_{1} v_{2} \ldots v_{t}$. We say that $T^{\prime}$ is obtained from $T$ by Operation I (as depicted in Figure 1).

Lemma 3.1 Let $T^{\prime}$ be obtained from $T$ by Operation $I$. Then $\mathcal{E}_{R}(T)<\mathcal{E}_{R}\left(T^{\prime}\right)$.

Proof. Let the $R$-characteristic polynomials of $T$ and $T^{\prime}$ be

$$
\phi_{R}(T, x)=\sum_{k=0}^{\left\lfloor\frac{n}{2}\right\rfloor}(-1)^{k} b(R(T), k) x^{n-2 k}, \quad \phi_{R}\left(T^{\prime}, x\right)=\sum_{k=0}^{\left\lfloor\frac{n}{2}\right\rfloor}(-1)^{k} b\left(R\left(T^{\prime}\right), k\right) x^{n-2 k},
$$




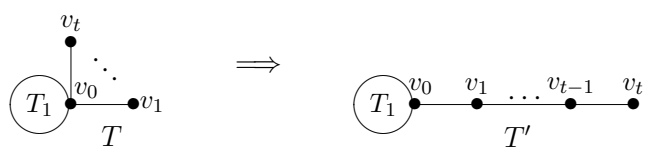

Figure 1. Operation I.

respectively, where $b(R(T), 0)=b\left(R\left(T^{\prime}\right), 0\right)=1$.

Denote $N_{T}\left(v_{0}\right)=\left\{v_{1}, \ldots, v_{t}, u_{1}, \ldots, u_{s}\right\}$, where $t \geq 2, s \geq 1$. Note that $d_{T}\left(v_{0}\right)=s+t$, $d_{T^{\prime}}\left(v_{0}\right)=s+1$, and $d_{T}\left(u_{i}\right)=d_{T^{\prime}}\left(u_{i}\right)$ for $i=1,2, \ldots, s$. Then

$$
\begin{aligned}
& b\left(R\left(T^{\prime}\right), 1\right)-b(R(T), 1) \\
= & \sum_{i=1}^{s} R_{T^{\prime}}\left(v_{0} u_{i}\right)+\sum_{j=0}^{t-1} R_{T^{\prime}}\left(v_{j} u_{j+1}\right)-\sum_{i=1}^{s} R_{T}\left(v_{0} u_{i}\right)-\sum_{j=1}^{t} R_{T}\left(v_{0} v_{j}\right) \\
= & \sum_{i=1}^{s} \frac{t-1}{(s+1)(s+t) d_{T}\left(u_{i}\right)}+\frac{s(t-1)(t-2)+t\left(s^{2}+t-2\right)}{4(s+1)(s+t)}>0 .
\end{aligned}
$$

For $2 \leq k \leq\left\lfloor\frac{n}{2}\right\rfloor$,

$$
\begin{aligned}
& b\left(R\left(T^{\prime}\right), k\right) \\
\geq & \sum_{\alpha_{k} \in \mathcal{M}_{k}\left(T_{1}\right)} R_{T}\left(\alpha_{k}\right)+\sum_{i=0}^{t-1} R_{T^{\prime}}\left(v_{i} v_{i+1}\right) \sum_{\alpha_{k-1} \in \mathcal{M}_{k-1}\left(T-v_{0}\right)} R_{T^{\prime}}\left(\alpha_{k-1}\right) \\
= & \sum_{\alpha_{k} \in \mathcal{M}_{k}\left(T_{1}\right)} R_{T}\left(\alpha_{k}\right)+\left(\frac{1}{2(s+1)}+\frac{t-2}{4}+\frac{1}{2}\right) \sum_{\alpha_{k-1} \in \mathcal{M}_{k-1}\left(T_{1}-v_{0}\right)} R_{T}\left(\alpha_{k-1}\right), \\
& b(R(T), k) \\
= & \sum_{\alpha_{k} \in \mathcal{M}_{k}\left(T_{1}\right)} R_{T}\left(\alpha_{k}\right)+\frac{t}{s+t} \sum_{\alpha_{k-1} \in \mathcal{M}_{k-1}\left(T-v_{0}\right)} R_{T}\left(\alpha_{k-1}\right), \\
& b\left(R\left(T^{\prime}\right), k\right)-b(R(T), k) \sum_{\sum_{k-1} \in \mathcal{M}_{k-1}\left(T-v_{0}\right)} R_{T}\left(\alpha_{k-1}\right)>0 .
\end{aligned}
$$

By Lemma 2.1, the lemma holds.

Corollary 3.2 Let $T \in \mathcal{T}(n)$ be a tree of order $n$ (as depicted in Figure 2(a)), where $T_{1}$ is a subtree of $T$ with $v_{0}, u_{0} \in V\left(T_{1}\right), t \geq 2, s \geq 2$. $T^{\prime}$ (as depicted in Figure 2(b)) is obtained from $T$ by Operation $I$, and $T^{\prime \prime}$ (as depicted in Figure 2(c)) is obtained from $T^{\prime}$ by Operation I. Then

$$
\mathcal{E}_{R}(T)<\mathcal{E}_{R}\left(T^{\prime}\right)<\mathcal{E}_{R}\left(T^{\prime \prime}\right)
$$




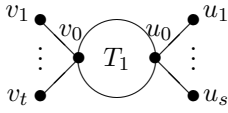

Figure 2(a). T

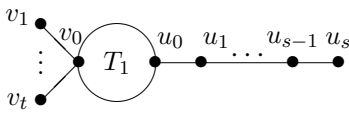

Figure 2(b). $T^{\prime}$

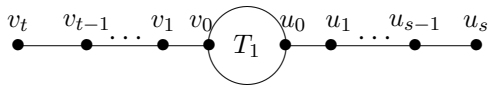

Figure 2(c). $T^{\prime \prime}$

By Corollary 3.2, the following result is clear.

Lemma 3.3 Let $T \in \mathcal{T}(n)$ and $u_{0}$ be a quasi-pendent vertex of $T$. If $d\left(u_{0}\right) \geq 3$ and there are at least two pendent vertices in $N_{T}\left(u_{0}\right)$, then there is $T^{\prime} \in \mathcal{T}(n)$ such that $T^{\prime}$ satisfies the following conditions.

(1) $u_{0}$ is no longer a quasi-pendent vertex and $u_{s-1}$ is a new small quasi-pendent vertex;

(2) There is just one pendent vertex in $N_{T^{\prime}}\left(u_{s-1}\right)$;

(3) $\mathcal{E}_{R}(T) \leq \mathcal{E}_{R}\left(T^{\prime}\right)$

Let $T$ be the tree as shown in Figure 2(c), where $T_{1}$ is a subtree of $T$ with $u_{1} \in V\left(T_{1}\right)$ and $d_{T}\left(u_{1}\right) \geq 2, t \geq 7$. Let $T^{\prime}=T-v_{4} v_{5} \ldots v_{t}+v_{1} v_{4} v_{5} \ldots v_{t}$ (as depicted in Figure 3 ). We say that $T^{\prime}$ is obtained from $T$ by Operation II for $v_{1}$.

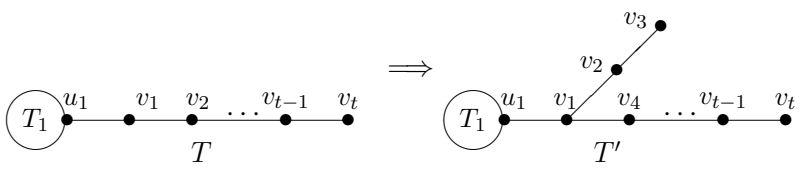

Figure 3. Operation II.

Lemma 3.4 Let $T^{\prime}$ be obtained from $T$ by Operation II. Then $\mathcal{E}_{R}(T) \leq \mathcal{E}_{R}\left(T^{\prime}\right)$.

Proof. Let the Randić characteristic polynomials of $T$ and $T^{\prime}$ be

$$
\phi_{R}(T, x)=\sum_{k=0}^{\left\lfloor\frac{n}{2}\right\rfloor}(-1)^{k} b(R(T), k) x^{n-2 k}, \quad \phi_{R}\left(T^{\prime}, x\right)=\sum_{k=0}^{\left\lfloor\frac{n}{2}\right\rfloor}(-1)^{k} b\left(R\left(T^{\prime}\right), k\right) x^{n-2 k},
$$

respectively, where $b(R(T), 0)=b\left(R\left(T^{\prime}\right), 0\right)=1$. 
Note that $d_{T}\left(v_{1}\right)=2, d_{T^{\prime}}\left(v_{1}\right)=3, d_{T}\left(v_{3}\right)=2, d_{T^{\prime}}\left(v_{3}\right)=1$ and $d_{T}\left(u_{1}\right)=d_{T^{\prime}}\left(u_{1}\right) \geq 2$. Then

$$
\begin{aligned}
& b\left(R\left(T^{\prime}\right), 1\right)-b(R(T), 1) \\
= & R_{T^{\prime}}\left(u_{1} v_{1}\right)+R_{T^{\prime}}\left(v_{1} v_{2}\right)+R_{T^{\prime}}\left(v_{2} v_{3}\right)+R_{T^{\prime}}\left(v_{1} v_{4}\right) \\
& -\left(R_{T}\left(u_{1} v_{1}\right)+R_{T}\left(v_{1} v_{2}\right)+R_{T}\left(v_{2} v_{3}\right)+R_{T}\left(v_{3} v_{4}\right)\right) \\
= & \frac{1}{12}-\frac{1}{6 d_{T}\left(u_{1}\right)} \geq 0 .
\end{aligned}
$$

For $2 \leq k \leq\left\lfloor\frac{n}{2}\right\rfloor$, denote $P=v_{5} v_{6} \ldots v_{t}$. Then

$$
\begin{aligned}
& b(M(T), k) \\
& =\sum_{\alpha_{k} \in \mathcal{M}_{k}\left(T_{1} \cup P\right)} R_{T}\left(\alpha_{k}\right)+\frac{1}{2 d_{T}\left(u_{1}\right)} \sum_{\alpha_{k-1} \in \mathcal{M}_{k-1}\left(\left(T_{1}-u_{1}\right) \cup P\right)} R_{T}\left(\alpha_{k-1}\right) \\
& +\frac{3}{4} \sum_{\alpha_{k-1} \in \mathcal{M}_{k-1}\left(T_{1} \cup P\right)} R_{T}\left(\alpha_{k-1}\right)+\frac{1}{4} \sum_{\alpha_{k-1} \in \mathcal{M}_{k-1}\left(T_{1} \cup\left(P-v_{5}\right)\right)} R_{T}\left(\alpha_{k-1}\right) \\
& +\frac{1}{4 d_{T}\left(u_{1}\right)} \sum_{\alpha_{k-2} \in \mathcal{M}_{k-2}\left(\left(T_{1}-u_{1}\right) \cup P\right)} R_{T}\left(\alpha_{k-2}\right) \\
& +\frac{1}{8 d_{T}\left(u_{1}\right)} \sum_{\alpha_{k-2} \in \mathcal{M}_{k-2}\left(\left(T_{1}-u_{1}\right) \cup\left(P-v_{5}\right)\right)} R_{T}\left(\alpha_{k-2}\right) \\
& +\frac{1}{16} \sum_{\alpha_{k-2} \in \mathcal{M}_{k-2}\left(T_{1} \cup P\right)} R_{T}\left(\alpha_{k-2}\right)+\frac{1}{8} \sum_{\alpha_{k-2} \in \mathcal{M}_{k-2}\left(T_{1} \cup\left(P-v_{5}\right)\right)} R_{T}\left(\alpha_{k-2}\right) \\
& +\frac{1}{32 d_{T}\left(u_{1}\right)} \sum_{\alpha_{k-3} \in \mathcal{M}_{k-3}\left(\left(T_{1}-u_{1}\right) \cup\left(P-v_{5}\right)\right)} R_{T}\left(\alpha_{k-3}\right), \\
& b\left(M\left(T^{\prime}\right), k\right) \\
& =\sum_{\alpha_{k} \in \mathcal{M}_{k}\left(T_{1} \cup P\right)} R_{T}\left(\alpha_{k}\right)+\frac{1}{3 d_{T}\left(u_{1}\right)} \sum_{\alpha_{k-1} \in \mathcal{M}_{k-1}\left(\left(T_{1}-u_{1}\right) \cup P\right)} R_{T}\left(\alpha_{k-1}\right) \\
& +\frac{5}{6} \sum_{\alpha_{k-1} \in \mathcal{M}_{k-1}\left(T_{1} \cup P\right)} R_{T}\left(\alpha_{k-1}\right)+\frac{1}{4} \sum_{\alpha_{k-1} \in \mathcal{M}_{k-1}\left(T_{1} \cup\left(P-v_{5}\right)\right)} R_{T}\left(\alpha_{k-1}\right) \\
& +\frac{1}{6 d_{T}\left(u_{1}\right)} \sum_{\alpha_{k-2} \in \mathcal{M}_{k-2}\left(\left(T_{1}-u_{1}\right) \cup P\right)} R_{T}\left(\alpha_{k-2}\right) \\
& +\frac{1}{12 d_{T}\left(u_{1}\right)} \sum_{\alpha_{k-2} \in \mathcal{M}_{k-2}\left(\left(T_{1}-u_{1}\right) \cup\left(P-v_{5}\right)\right)} R_{T}\left(\alpha_{k-2}\right) \\
& +\frac{1}{12} \sum_{\alpha_{k-2} \in \mathcal{M}_{k-2}\left(T_{1} \cup P\right)} R_{T}\left(\alpha_{k-2}\right)+\frac{1}{6} \sum_{\alpha_{k-2} \in \mathcal{M}_{k-2}\left(T_{1} \cup\left(P-v_{5}\right)\right)} R_{T}\left(\alpha_{k-2}\right) \\
& +\frac{1}{24 d_{T}\left(u_{1}\right)} \sum_{\alpha_{k-3} \in \mathcal{M}_{k-3}\left(\left(T_{1}-u_{1}\right) \cup\left(P-v_{5}\right)\right)} R_{T}\left(\alpha_{k-3}\right) .
\end{aligned}
$$


Note that

$$
\begin{aligned}
& \frac{1}{12} \sum_{\alpha_{k-1} \in \mathcal{M}_{k-1}\left(T_{1} \cup P\right)} R_{T}\left(\alpha_{k-1}\right) \geq \frac{1}{6 d_{T}\left(u_{1}\right)} \sum_{\alpha_{k-1} \in \mathcal{M}_{k-1}\left(\left(T_{1}-u_{1}\right) \cup P\right)} R_{T}\left(\alpha_{k-1}\right), \\
& \sum_{\alpha_{k-2} \in \mathcal{M}_{k-2}\left(T_{1} \cup P\right)} R_{T}\left(\alpha_{k-2}\right) \\
& =\sum_{\alpha_{k-2} \in \mathcal{M}_{k-2}\left(T_{1} \cup\left(P-v_{5}\right)\right)} R_{T}\left(\alpha_{k-2}\right)+\frac{1}{4} \sum_{\alpha_{k-3} \in \mathcal{M}_{k-3}\left(T_{1} \cup\left(P-v_{5}-v_{6}\right)\right)} R_{T}\left(\alpha_{k-3}\right), \\
& \sum_{\alpha_{k-2} \in \mathcal{M}_{k-2}\left(\left(T_{1}-u_{1}\right) \cup P\right)} R_{T}\left(\alpha_{k-2}\right) \\
& =\sum_{\alpha_{k-2} \in \mathcal{M}_{k-2}\left(\left(T_{1}-u_{1}\right) \cup\left(P-v_{5}\right)\right)} R_{T}\left(\alpha_{k-2}\right)+\frac{1}{4} \sum_{\alpha_{k-3} \in \mathcal{M}_{k-3}\left(\left(T_{1}-u_{1}\right) \cup\left(P-v_{5}-v_{6}\right)\right)} R_{T}\left(\alpha_{k-3}\right) .
\end{aligned}
$$

So

$$
\begin{aligned}
& b\left(M\left(T^{\prime}\right), k\right)-b(M(T), k) \\
& =-\frac{1}{6 d_{T}\left(u_{1}\right)} \sum_{\alpha_{k-1} \in \mathcal{M}_{k-1}\left(\left(T_{1}-u_{1}\right) \cup P\right)} R_{T}\left(\alpha_{k-1}\right)+\frac{1}{12} \sum_{\alpha_{k-1} \in \mathcal{M}_{k-1}\left(T_{1} \cup P\right)} R_{T}\left(\alpha_{k-1}\right) \\
& -\frac{1}{12 d_{T}\left(u_{1}\right)} \sum_{\alpha_{k-2} \in \mathcal{M}_{k-2}\left(\left(T_{1}-u_{1}\right) \cup P\right)} R_{T}\left(\alpha_{k-2}\right) \\
& -\frac{1}{24 d_{T}\left(u_{1}\right)} \sum_{\alpha_{k-2} \in \mathcal{M}_{k-2}\left(\left(T_{1}-u_{1}\right) \cup\left(P-v_{5}\right)\right)} R_{T}\left(\alpha_{k-2}\right) \\
& +\frac{1}{48} \sum_{\alpha_{k-2} \in \mathcal{M}_{k-2}\left(T_{1} \cup P\right)} R_{T}\left(\alpha_{k-2}\right)+\frac{1}{24} \sum_{\alpha_{k-2} \in \mathcal{M}_{k-2}\left(T_{1} \cup\left(P-v_{5}\right)\right)} R_{T}\left(\alpha_{k-2}\right) \\
& +\frac{1}{96 d_{T}\left(u_{1}\right)} \sum_{\alpha_{k-3} \in \mathcal{M}_{k-3}\left(\left(T_{1}-u_{1}\right) \cup\left(P-v_{5}\right)\right)} R_{T}\left(\alpha_{k-3}\right) \\
& \geq-\frac{1}{12 d_{T}\left(u_{1}\right)} \sum_{\alpha_{k-2} \in \mathcal{M}_{k-2}\left(\left(T_{1}-u_{1}\right) \cup\left(P-v_{5}\right)\right)} R_{T}\left(\alpha_{k-2}\right) \\
& -\frac{1}{12 d_{T}\left(u_{1}\right)} \times \frac{1}{4} \sum_{\alpha_{k-3} \in \mathcal{M}_{k-3}\left(\left(T_{1}-u_{1}\right) \cup\left(P-v_{5}-v_{6}\right)\right)} R_{T}\left(\alpha_{k-3}\right) \\
& -\frac{1}{24 d_{T}\left(u_{1}\right)} \sum_{\alpha_{k-2} \in \mathcal{M}_{k-2}\left(\left(T_{1}-u_{1}\right) \cup\left(P-v_{5}\right)\right)} R_{T}\left(\alpha_{k-2}\right) \\
& +\frac{1}{48} \sum_{\alpha_{k-2} \in \mathcal{M}_{k-2}\left(T_{1} \cup\left(P-v_{5}\right)\right)} R_{T}\left(\alpha_{k-2}\right)+\frac{1}{48} \times \frac{1}{4} \sum_{\alpha_{k-3} \in \mathcal{M}_{k-3}\left(T_{1} \cup\left(P-v_{5}-v_{6}\right)\right)} R_{T}\left(\alpha_{k-3}\right)
\end{aligned}
$$




$$
\begin{aligned}
& +\frac{1}{24} \sum_{\alpha_{k-2} \in \mathcal{M}_{k-2}\left(T_{1} \cup\left(P-v_{5}\right)\right)} R_{T}\left(\alpha_{k-2}\right)+\frac{1}{96 d_{T}\left(u_{1}\right)} \sum_{\alpha_{k-3} \in \mathcal{M}_{k-3}\left(\left(T_{1}-u_{1}\right) \cup\left(P-v_{5}\right)\right)} R_{T}\left(\alpha_{k-3}\right) \\
& =\frac{1}{16} \sum_{\alpha_{k-2} \in \mathcal{M}_{k-2}\left(T_{1} \cup\left(P-v_{5}\right)\right)} R_{T}\left(\alpha_{k-2}\right)-\frac{1}{8 d_{T}\left(u_{1}\right)} R_{\alpha_{k-2} \in \mathcal{M}_{k-2}\left(\left(T_{1}-u_{1}\right) \cup\left(P-v_{5}\right)\right)}\left(\alpha_{k-2}\right) \\
& +\frac{1}{192} \sum_{\alpha_{k-3} \in \mathcal{M}_{k-3}\left(T_{1} \cup\left(P-v_{5}-v_{6}\right)\right)} R_{T}\left(\alpha_{k-3}\right) \\
& -\frac{1}{96 d_{T}\left(u_{1}\right)} \sum_{\alpha_{k-3} \in \mathcal{M}_{k-3}\left(\left(T_{1}-u_{1}\right) \cup\left(P-v_{5}-v_{6}\right)\right)} R_{T}\left(\alpha_{k-3}\right) \\
& -\frac{1}{96 d_{T}\left(u_{1}\right)} \sum_{\alpha_{k-3} \in \mathcal{M}_{k-3}\left(\left(T_{1}-u_{1}\right) \cup\left(P-v_{5}-v_{6}\right)\right)} R_{T}\left(\alpha_{k-3}\right) \\
& +\frac{1}{96 d_{T}\left(u_{1}\right)} \\
& \sum_{\alpha_{k-3} \in \mathcal{M}_{k-3}\left(\left(T_{1}-u_{1}\right) \cup\left(P-v_{5}\right)\right)} R_{T}\left(\alpha_{k-3}\right) .
\end{aligned}
$$

Since $d_{T}\left(u_{1}\right) \geq 2$, and

$$
\begin{aligned}
\sum_{\alpha_{k-2} \in \mathcal{M}_{k-2}\left(T_{1} \cup\left(P-v_{5}\right)\right)} R_{T}\left(\alpha_{k-2}\right) & \geq \sum_{\alpha_{k-2} \in \mathcal{M}_{k-2}\left(\left(T_{1}-u_{1}\right) \cup\left(P-v_{5}\right)\right)} R_{T}\left(\alpha_{k-2}\right), \\
\sum_{\alpha_{k-3} \in \mathcal{M}_{k-3}\left(T_{1} \cup\left(P-v_{5}-v_{6}\right)\right)} R_{T}\left(\alpha_{k-3}\right) & \geq \sum_{\alpha_{k-3} \in \mathcal{M}_{k-3}\left(\left(T_{1}-u_{1}\right) \cup\left(P-v_{5}-v_{6}\right)\right)} R_{T}\left(\alpha_{k-3}\right), \\
\sum_{\alpha_{k-3} \in \mathcal{M}_{k-3}\left(\left(T_{1}-u_{1}\right) \cup\left(P-v_{5}\right)\right)} R_{T}\left(\alpha_{k-3}\right) & \geq \sum_{\alpha_{k-3} \in \mathcal{M}_{k-3}\left(\left(T_{1}-u_{1}\right) \cup\left(P-v_{5}-v_{6}\right)\right)} R_{T}\left(\alpha_{k-3}\right),
\end{aligned}
$$

we get $b\left(M\left(T^{\prime}\right), k\right)-b(M(T), k) \geq 0$.

By Lemma $2.1, \mathcal{E}_{R}(T) \leq \mathcal{E}_{R}\left(T^{\prime}\right)$.

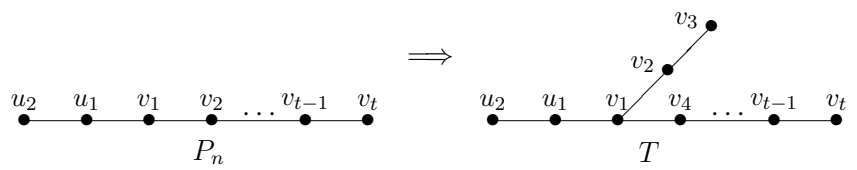

Figure 4 .

Corollary 3.5 Let $T$ be the tree of order $n \geq 9$ (as depicted in Figure 4). Then

$$
\mathcal{E}_{R}\left(P_{n}\right) \leq \mathcal{E}_{R}(T)
$$

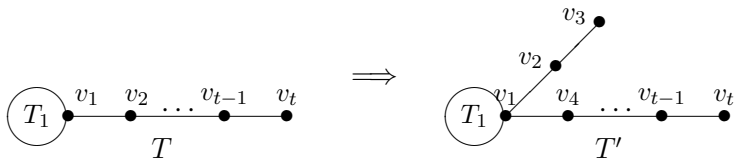

Figure 5. Operation III. 
Lemma 3.6 ( $[8])$ Let $T$ and $T^{\prime}$ be trees of order $n$ as depicted in Figure 5, where $t \geq 5$, $d_{T}\left(v_{1}\right) \geq 3, N_{T}\left(v_{1}\right)$ has no pendent vertices, and $T_{1}$ is a subtree of $T$ with $v_{1} \in V\left(T_{1}\right)$. Then $\mathcal{E}_{R}(T) \leq \mathcal{E}_{R}\left(T^{\prime}\right)$.

\section{Main result}

In this section, we will show that for any coral tree $T \in \mathcal{T}(n), \mathcal{E}_{R}(T) \leq \mathcal{E}_{R}\left(P_{n}\right)$. That is, $P_{n}$ is the connected $n$-vertex graph with maximal Randić energy for $\mathcal{T}(n)$. Furthermore, we will prove that if for a graph $G, \mathcal{E}_{R}(G) \leq \mathcal{E}_{R}\left(P_{n}\right)$, then $G$ satisfies Conjecture 1.1. That means that the coral trees set $T(n)$ is a family that satisfies Conjecture 1.1.

Lemma 4.1 Let $T \in \mathcal{T}(n)$ be a coral tree with two small quasi-pendent vertices. If there is just one pendent vertex in the neighborhood of a small quasi-pendent vertex, then $\mathcal{E}_{R}(T) \leq \mathcal{E}_{R}\left(P_{n}\right)$

Proof. Without loss of generality, we assume that $v_{2}, v_{n-1}$ are two small quasi-pendent vertices. Then $T$ is a tree as depicted in Figure 6, where $T_{1}$ is a subtree of order $n-2$. Consider the tree $T^{\prime}$ which is obtained from $T$ by replacing $T_{1}$ with the path of order $n-2$. Clearly, $T^{\prime}$ is a path of order $n$ as depicted in Figure 6. For $1 \leq i<j \leq n$, use $P_{v_{i}, v_{j}}$ to denote the path of $T^{\prime}$ from $v_{i}$ to $v_{j}$.

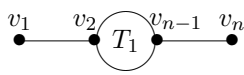

T

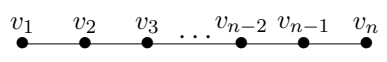

$T^{\prime}$

Figure 6.

Let the Randic characteristic polynomials of $T$ and $T^{\prime}$ be

$$
\phi_{R}(T, x)=\sum_{k=0}^{\left\lfloor\frac{n}{2}\right\rfloor}(-1)^{k} b(R(T), k) x^{n-2 k}, \quad \phi_{R}\left(T^{\prime}, x\right)=\sum_{k=0}^{\left\lfloor\frac{n}{2}\right\rfloor}(-1)^{k} b\left(R\left(T^{\prime}\right), k\right) x^{n-2 k},
$$

respectively, where $b(R(T), 0)=b\left(R\left(T^{\prime}\right), 0\right)=1$.

Note that the following facts.

- $R_{T^{\prime}}\left(v_{1} v_{2}\right)=\frac{1}{2} \geq R_{T}\left(v_{1} v_{2}\right), R_{T^{\prime}}\left(v_{n-1} v_{n}\right)=\frac{1}{2} \geq R_{T}\left(v_{n-1} v_{n}\right)$.

- For each edge $v_{i} v_{j} \in E\left(T^{\prime}\right), 2 \leq i<j \leq n-1, R_{T^{\prime}}\left(v_{i} v_{j}\right)=\frac{1}{4}$. For each edge $u v \in E(T) \backslash\left\{v_{1} v_{2}, v_{n-1} v_{n}\right\}, R_{T}(u v) \leq \frac{1}{4}$. 
- For any positive integer $l$, by Lemma 2.2,

$$
\begin{aligned}
\sum_{\alpha_{l}^{\prime} \in \mathcal{M}_{l}\left(P_{v_{2}, v_{n-1}}\right)} R_{T^{\prime}}\left(\alpha_{l}^{\prime}\right)=\left(\frac{1}{4}\right)^{l}\left|\mathcal{M}_{l}\left(P_{v_{2}, v_{n-1}}\right)\right| & \geq \sum_{\alpha_{l} \in \mathcal{M}_{l}\left(T_{1}\right)} R_{T}\left(\alpha_{l}\right), \\
\sum_{\alpha_{l}^{\prime} \in \mathcal{M}_{l}\left(P_{v_{3}, v_{n-1}}\right)} R_{T^{\prime}}\left(\alpha_{l}^{\prime}\right)=\left(\frac{1}{4}\right)^{l}\left|\mathcal{M}_{l}\left(P_{v_{3}, v_{n-1}}\right)\right| & \geq \sum_{\alpha_{l} \in \mathcal{M}_{l}\left(T_{1}-v_{2}\right)} R_{T}\left(\alpha_{l}\right), \\
\sum_{\alpha_{l}^{\prime} \in \mathcal{M}_{l}\left(P_{v_{2}, v_{n-2}}\right)} R_{T^{\prime}}\left(\alpha_{l}^{\prime}\right)=\left(\frac{1}{4}\right)^{l}\left|\mathcal{M}_{l}\left(P_{v_{2}, v_{n-2}}\right)\right| & \geq \sum_{\alpha_{l} \in \mathcal{M}_{l}\left(T_{1}-v_{n-1}\right)} R_{T}\left(\alpha_{l}\right), \\
\sum_{\alpha_{l}^{\prime} \in \mathcal{M}_{l}\left(P_{v_{3}, v_{n-2}}\right)} R_{T^{\prime}}\left(\alpha_{l}^{\prime}\right)=\left(\frac{1}{4}\right)^{l}\left|\mathcal{M}_{l}\left(P_{v_{3}, v_{n-2}}\right)\right| & \geq \sum_{\alpha_{l} \in \mathcal{M}_{l}\left(T_{1}-v_{2}-v_{n-1}\right)} R_{T}\left(\alpha_{l}\right) .
\end{aligned}
$$

It is clear that $b\left(R\left(T^{\prime}\right), k\right) \geq b(R(T), k)$ for $2 \leq k \leq\left\lfloor\frac{n}{2}\right\rfloor$.

By Lemma 2.1, $\mathcal{E}_{R}(T) \leq \mathcal{E}_{R}\left(T^{\prime}\right)=\mathcal{E}_{R}\left(P_{n}\right)$.

Theorem 4.2 Let $T \in \mathcal{T}(n)$ be a coral tree. Then $\mathcal{E}_{R}(T) \leq \mathcal{E}_{R}\left(P_{n}\right)$.

Proof. If $T \in \mathcal{T}(n)$ is a coral tree with two small quasi-pendent vertices and there is just one pendent vertex in the neighborhood of a small quasi-pendent vertex, then by Lemma $4.1, \mathcal{E}_{R}(T) \leq \mathcal{E}_{R}\left(P_{n}\right)$.

Otherwise, by Lemma 3.3, there is $T^{\prime} \in \mathcal{T}(n)$ satisfies the following conditions.

(1) $T^{\prime} \in \mathcal{T}(n)$ is a coral tree with two small quasi-pendent vertices;

(2) There is just one pendent vertex in the neighborhood of a small quasi-pendent vertex;

(3) $\mathcal{E}_{R}(T) \leq \mathcal{E}_{R}\left(T^{\prime}\right)$.

Therefore $\mathcal{E}_{R}(T) \leq \mathcal{E}_{R}\left(T^{\prime}\right) \leq \mathcal{E}_{R}\left(P_{n}\right)$.

Theorem 4.3 Let $n$ be odd. Then $\mathcal{E}_{R}\left(P_{n}\right) \leq \mathcal{E}_{R}\left(S u_{\frac{n-1}{2}}\right)$.

Proof. Case 1. $3 \leq n \leq 7$.

If $n=3$, then $P_{3} \cong S u_{1}$.

If $n=5$, then $P_{5} \cong S u_{2}$.

If $n=7$, we denote $T=S u_{3}$ and

$$
\phi_{R}(T, x)=\sum_{k=0}^{\left\lfloor\frac{7}{2}\right\rfloor}(-1)^{k} b(R(T), k) x^{n-2 k}, \quad \phi_{R}\left(P_{7}, x\right)=\sum_{k=0}^{\left\lfloor\frac{7}{2}\right\rfloor}(-1)^{k} b\left(R\left(P_{7}\right), k\right) x^{n-2 k},
$$


where $b(R(T), 0)=b\left(R\left(P_{7}\right), 0\right)=1$. Note that

$$
\begin{aligned}
& b(M(T), 1)-b\left(M\left(P_{7}\right), 1\right)=2-2=0, \\
& b(M(T), 2)-b\left(M\left(P_{7}\right), 2\right)=\frac{5}{4}-\frac{19}{16}>0, \\
& b(M(T), 3)-b\left(M\left(P_{7}\right), 3\right)=\frac{1}{4}-\frac{3}{16}>0 .
\end{aligned}
$$

By Lemma 2.1, $\mathcal{E}_{R}\left(P_{7}\right)<\mathcal{E}_{R}(T)$.

Case 2. $n \geq 9$.

Let $T$ be the tree as depicted in Figure 4. By Corollary 3.5, $\mathcal{E}_{R}\left(P_{n}\right) \leq \mathcal{E}_{R}(T)$. Applying Operation III to $T$ successively, by Lemma 3.6, we find that the Randić energy is increasing as $d_{T}\left(v_{1}\right)$ is increasing. So, $\mathcal{E}_{R}\left(P_{n}\right) \leq \mathcal{E}_{R}\left(S u_{\frac{n-1}{2}}\right)$.

The theorem now follows.

Theorem 4.4 Let $n$ be even. Then $\mathcal{E}_{R}\left(P_{n}\right) \leq \mathcal{E}_{R}\left(D S u_{\left\lfloor\frac{n-2}{4}\right\rfloor,\left\lceil\frac{n-2}{4}\right\rceil}\right)$.

Proof. Case 1. $2 \leq n \leq 12$.

If $n=2$, then $P_{2} \cong D S u_{0,0}$.

If $n=4$, then $P_{4} \cong D S u_{0,1}$.

If $n=6$, then $P_{6} \cong D S u_{1,1}$.

If $n=8$, we denote $T=D S u_{1,2}$ and

$$
\phi_{R}(T, x)=\sum_{k=0}^{4}(-1)^{k} b(R(T), k) x^{n-2 k}, \quad \phi_{R}\left(P_{8}, x\right)=\sum_{k=0}^{4}(-1)^{k} b\left(R\left(P_{8}\right), k\right) x^{n-2 k},
$$

where $b(R(T), 0)=b\left(R\left(P_{8}\right), 0\right)=1$. Note that

$$
\begin{aligned}
& b\left(M((T), 1)-b\left(M\left(P_{8}\right), 1\right)=\frac{9}{4}-\frac{9}{4}=0,\right. \\
& b(M(T), 2)-b\left(M\left(P_{8}\right), 2\right)=\frac{5}{3}-\frac{13}{8}>0, \\
& b(M(T), 3)-b\left(M\left(P_{8}\right), 3\right)=\frac{7}{16}-\frac{25}{64}>0, \\
& b(M(T), 4)-b\left(M\left(P_{8}\right), 4\right)=\frac{1}{48}-\frac{1}{64}>0 .
\end{aligned}
$$

By Lemma 2.1, $\mathcal{E}_{R}\left(P_{8}\right)<\mathcal{E}_{R}\left(D S u_{1,2}\right)$.

If $n=10$, we denote $T=D S u_{2,2}$ and

$$
\phi_{R}(T, x)=\sum_{k=0}^{5}(-1)^{k} b(R(T), k) x^{n-2 k}, \quad \phi_{R}\left(P_{10}, x\right)=\sum_{k=0}^{5}(-1)^{k} b\left(R\left(P_{10}\right), k\right) x^{n-2 k},
$$


where $b(R(T), 0)=b\left(R\left(P_{10}\right), 0\right)=1$. Note that

$$
\begin{aligned}
& b(M(T), 1)-b\left(M\left(P_{10}\right), 1\right)=\frac{25}{9}-\frac{11}{4}>0, \\
& b(M(T), 2)-b\left(M\left(P_{10}\right), 2\right)=\frac{17}{6}-\frac{43}{16}>0, \\
& b(M(T), 3)-b\left(M\left(P_{10}\right), 3\right)=\frac{23}{18}-\frac{35}{32}>0, \\
& b(M(T), 4)-b\left(M\left(P_{10}\right), 4\right)=\frac{25}{144}-\frac{41}{256}>0, \\
& b(M(T), 5)-b\left(M\left(P_{10}\right), 5\right)=\frac{1}{144}-\frac{1}{256}>0 .
\end{aligned}
$$

By Lemma 2.1, $\mathcal{E}_{R}\left(P_{10}\right)<\mathcal{E}_{R}\left(D S u_{2,2}\right)$.

If $n=12$, we denote $T=D S u_{2,3}$ and

$$
\phi_{R}(T, x)=\sum_{k=0}^{6}(-1)^{k} b(R(T), k) x^{n-2 k}, \quad \phi_{R}\left(P_{12}, x\right)=\sum_{k=0}^{6}(-1)^{k} b\left(R\left(P_{12}\right), k\right) x^{n-2 k},
$$

where $b(R(T), 0)=b\left(R\left(P_{12}\right), 0\right)=1$. Note that

$$
\begin{aligned}
& b(M(T), 1)-b\left(M\left(P_{12}\right), 1\right)=\frac{79}{24}-\frac{13}{4}=\frac{1}{24}>0, \\
& b(M(T), 2)-b\left(M\left(P_{12}\right), 2\right)=\frac{101}{24}-4>0 \\
& b(M(T), 3)-b\left(M\left(P_{12}\right), 3\right)=\frac{251}{72}-\frac{133}{64}>0, \\
& b(M(T), 4)-b\left(M\left(P_{12}\right), 4\right)=\frac{83}{96}-\frac{139}{256}>0 \\
& b(M(T), 5)-b\left(M\left(P_{12}\right), 5\right)=\frac{17}{192}-\frac{39}{1024}>0, \\
& b(M(T), 6)-b\left(M\left(P_{12}\right), 6\right)=\frac{1}{384}-\frac{1}{1024}>0 .
\end{aligned}
$$

By Lemma 2.1, $\mathcal{E}_{R}\left(P_{12}\right)<\mathcal{E}_{R}\left(D S u_{2,3}\right)$.

Case 2. $n \geq 14$.

Denote by $T^{\prime}$ and $T^{\prime \prime}$ the trees depicted in Figures $7(\mathrm{a})$ and $7(\mathrm{~b})$, where $x=\frac{n}{2}$ if $\frac{n}{2}$ is odd, and $x=\frac{n-2}{2}$ if $\frac{n}{2}$ is even.

Let $T$ be the tree of order $n \geq 14$ as depicted in Figure 4. By Corollary 3.5, $\mathcal{E}_{R}\left(P_{n}\right)<$ $\mathcal{E}_{R}(T)$. Applying Operation III to $T$ successively, by Lemma 3.6, $\mathcal{E}_{R}\left(P_{n}\right)<\mathcal{E}_{R}\left(T^{\prime}\right)$.

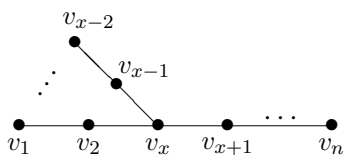

Figure 7(a). Tree $T^{\prime}$

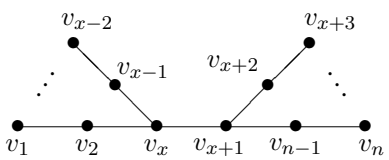

Figure $7(\mathbf{b})$. Tree $T^{\prime \prime}$ 
Applying Operation II to $T^{\prime}$ for $v_{x+1}$ firstly, then applying Operation III to the resulting tree successively, by Lemmas 3.4 and 3.6, we find that the Randić energy is increasing as $d_{T}\left(v_{x+1}\right)$ is increasing. So, $\mathcal{E}_{R}\left(P_{n}\right)<\mathcal{E}_{R}\left(T^{\prime}\right)<\mathcal{E}_{R}\left(T^{\prime \prime}\right)$, where $T^{\prime \prime}$ is $D S u_{\left\lfloor\frac{n-2}{4}\right\rfloor,\left\lceil\frac{n-2}{4}\right\rceil}$ as depicted in Figure $7(\mathrm{~b})$.

By Theorems 4.2, 4.3 and 4.4, we get the following results.

(1) For $T \in \mathcal{T}(n), \mathcal{E}_{R}(T) \leq \mathcal{E}_{R}\left(P_{n}\right)$.

(2) For a graph $G$, if $\mathcal{E}_{R}(G) \leq \mathcal{E}_{R}\left(P_{n}\right)$, then $G$ satisfies Conjecture 1.1.

(3) $\mathcal{T}(n)$ is a family of trees that satisfies Conjecture 1.1.

\section{References}

[1] L. E. Allem, R. O. Braga, A. Pastine, Randić index and energy, MATCH Commun. Math. Comput. Chem. 83 (2020) 611-622.

[2] L. E. Allem, G. Molina, A. Pastine, Short note on Randić energy, MATCH Commun. Math. Comput. Chem. 82 (2019) 515-528.

[3] S. B. Bozkurt, A. D. Güngör, I. Gutman, Randić spectral radius and Randić energy, MATCH Commun. Math. Comput. Chem. 64 (2010) 321-334.

[4] S. B. Bozkurt, A. D. Güngör, I. Gutman, A. S. Cevik, Randić matrix and Randić energy, MATCH Commun. Math. Comput. Chem. 64 (2010) 239-250.

[5] D. M. Cvetković, M. Doob, H. Sachs, Spectra of Graphs - Theory and Applications, Barth Verlag, Heidelberg, 1995.

[6] K. C. Das, S. Sorgun, On Randić energy of graphs, MATCH Commun. Math. Comput. Chem. 72 (2014) 227-238.

[7] K. C. Das, S. Sorgun, I. Gutman, On Randić energy, MATCH Commun. Math. Comput. Chem. 73 (2015) 81-92.

[8] W. Gao, The Randić energy of generalized double sun, Czech. Math. J. (2021) https: //doi.org/10.21136/CMJ . 2021.0463-20.

[9] Y. Gao, W. Gao, Y. Shao, The minimal Randić energy of trees with given diameter, Appl. Math. Comput. 411 (2021) \#126489.

[10] I. Gutman, Acyclic systems with extremal Hückel $\pi$-electron energy, Theor. Chim. Acta 45 (1977) 79-87. 
[11] I. Gutman, B. Furtula, S. B. Bozkurt, On Randić energy, Lin. Algebra Appl. 442 (2014) $50-57$.

[12] J. He, Y. M. Liu, J. K. Tian, Note on the Randić energy of graphs, Kragujevac J. Math. 42 (2018) 209-215.

[13] M. Randić, On characterization of molecular branching, J. Am. Chem. Soc. 97 (1975) $6609-6615$. 